\title{
FURTHER RESULTS ON THE CONSTRUCTION OF MUTUALLY ORTHOGONAL LATIN SQUARES AND THE FALSITY OF EULER'S CONJECTURE
}

\author{
R. C. BOSE, S. S. SHRIKHANDE, AND E. T. PARKER
}

\section{Introduction. If}

$$
p_{1}^{n_{1}} p_{2}^{n_{2}} \ldots p_{u}^{n_{u}}
$$

is the prime power decomposition of an integer $v$, and we define the arithmetic function $n(v)$ by

$$
n(v)=\min \left(p_{1}^{n_{1}}, p_{2}^{n_{2}}, \ldots, p_{u}^{n_{u}}\right)-1,
$$

then it is known, MacNeish (10) and Mann (11), that there exists a set of at least $n(v)$ mutually orthogonal Latin squares (m.o.l.s.) of order $v$. We shall denote by $N(v)$ the maximum possible number of mutually orthogonal Latin squares of order $v$. Then the Mann-MacNeish theorem can be stated as

$$
N(v) \geqslant n(v) \text {. }
$$

MacNeish conjectured that the actual value of $N(v)$ is $n(v)$. This conjecture seemed plausible as it implied the correctness of Euler's conjecture $(8$, p. $383, \S 144)$ about the non-existence of two orthogonal Latin squares of order $v=4 t+2$ (since $n(v)=1$ in this case), and also the well-known result

$$
N(v)=n(v)=p^{m}-1 \text { when } v=p^{m} \text { and } p \text { is a prime. }
$$

MacNeish's conjecture was disproved by Parker (12) who showed that in certain cases $N(v)>n(v)$ by proving that if there exists a balanced incomplete block (BIB) design with $v$ treatments, $\lambda=1$, and block size $k$ which is a prime power then $N(v) \geqslant k-2$, and that this result can be improved to $N(v) \geqslant k-1$, when the design is symmetric and cyclic.

Parker's result though it did not disprove Euler's conjecture threw serious doubts on its correctness. Bose and Shrikhande (4) were able to obtain a counter example by using a general class of designs, viz., the pairwise balanced designs of index unity. They showed (6) that Euler's conjecture is false for an infinity of values of $v \geqslant 22$, and obtained improved lower bounds for $N(v)$ for a large class of values of $v$.

By using the method of differences Parker (13) showed that $N(v) \geqslant 2$ for

Received August 30, 1959. This research was supported in part by the United States Air Force through the Air Force Office of Scientific Research of the Air Research and Development Command, under Contract No. AF 49(638)-213. Reproduction in whole or in part is permitted for any purpose of the United States Government. 
$v=\frac{1}{2}(3 q-1)$, where $q$ is a prime power $\equiv 3(\bmod 4)$. This includes the case $v=10$.

In the present paper (i) the main theorem of (6) has been improved enabling us to obtain better bounds on $N(v)$, (ii) the method of differences has been used to show that $N(v) \geqslant 2$ when $v=14,26$, or $12 t+10$, and (iii) Euler's conjecture has been shown to be false for all $v=4 t+2>6$.

2. Definitions and notations. We shall try to adhere as much as possible to the notation and definitions used in (6).

A Latin square of order $v$ may be defined as an arrangement of $v$ symbols say, $1,2, \ldots, v$ in a $v \times v$ square such that each symbol occurs exactly once in every row and once in every column. Two Latin squares are said to be orthogonal if, when they are superposed, each symbol of the first square occurs just once with each symbol of the second square. A set of mutually orthogonal Latin squares is a set of Latin squares any two of which are orthogonal.

An orthogonal array $\left(k^{2}, q+1, k, 2\right)$ of size $k^{2}, q+1$ constraints, $k$ levels and strength 2 is a $k^{2} \times(q+1)$ matrix $A$ whose elements are $k$ symbols, such that every two-rowed submatrix of $A$ contains as a column vector every possible pair of symbols. It is well known $(7 ; \mathbf{1 4})$ that the existence of $q-1$ mutually orthogonal $k \times k$ Latin squares implies the existence of an orthogonal array $\left(k^{2}, q+1, k, 2\right)$ and conversely.

An arrangement of $v$ objects (called treatments) in $b$ sets (called blocks) will be called a pairwise balanced design of index unity and type $\left(v ; k_{1}, k_{2}, \ldots\right.$, $k_{m}$ ) if each block contains either $k_{1}, k_{2}, \ldots$, or $k_{m}$ treatments which are all distinct $\left(k_{i} \leqslant v, k_{i} \neq k_{j}\right)$, and every pair of distinct treatments occurs in exactly one block of the design. If the number of blocks containing $k_{i}$ treatments is $b_{i}$, then clearly

$$
b=\sum_{i=1}^{m} b_{i}, \quad v(v-1)=\sum_{i=1}^{m} b_{i} k_{i}\left(k_{i}-1\right) .
$$

Consider a pairwise balanced design $(D)$ of index unity and type $\left(v ; k_{1}\right.$, $\left.k_{2}, \ldots, k_{m}\right)$. The subdesign $\left(D_{i}\right)$ formed by the blocks of size $k_{i}$ will be called the $i$ th equiblock component of $(D), i=1,2, \ldots, m$.

A subset of blocks belonging to any equiblock component $\left(D_{i}\right)$ will be said to be of type I if every treatment occurs in the subset exactly $k_{i}$ times. The number of blocks in such a subset is clearly $v$. As noted by Levi using König's theorem on the decomposition of even regular graphs (9, pp. 4-6), we can rearrange the treatments within the blocks of the subset in such a way that every treatment comes in each position exactly once. If the $v$ blocks of the subset are written out as columns, each treatment occurs exactly once in every row. When so written out the blocks will be said to be in the standard form. A subset of blocks belonging to $\left(D_{i}\right)$ will be said to be of type II if every treatment occurs in the subset exactly once. The component $\left(D_{i}\right)$ will be defined to be separable if the blocks can be divided into subsets of type I or 
type II (both types may occur at the same time). The design $(D)$ is defined to be separable if each equiblock component is separable.

The set of equiblock components $\left(D_{1}\right),\left(D_{2}\right), \ldots,\left(D_{l}\right), l<m$, will be said to be a clear set if the $\sum_{i=1}^{l} b_{i}$ blocks comprising $\left(D_{1}\right),\left(D_{2}\right), \ldots,\left(D_{l}\right)$ are disjoint, that is, no two blocks contain a common treatment. Clearly a necessary condition for this is

$$
\sum_{i=1}^{l} b_{i} k_{i} \leqslant v
$$

We shall have occasion to use the following Lemmas proved in (6).

Lemma 1. If $v=v_{1} v_{2} \ldots v_{u}$ then $N(v) \geqslant \min \left(N\left(v_{1}\right), N\left(v_{2}\right), \ldots, N\left(v_{u}\right)\right)$.

Lemma 2. Suppose there exists a set $\sum$ of $q-1$ m.o.l.s. of order $k$, then we can construct a $q \times k(k-1)$ matrix $P$, whose elements are the symbols $1,2, \ldots$, $k$ and such that (i) any ordered pair

$$
\left(\begin{array}{l}
i \\
j
\end{array}\right), i \neq j
$$

occurs as a column exactly once in any two-rowed submatrix of $P$, (ii) $P$ can be subdivided into $k-1$ submatrices $P_{1}, P_{2}, \ldots, P_{k-1}$ of order $q \times k$ such that in each row of $P_{c}, 1 \leqslant c \leqslant k-1$, each of the symbols $1,2, \ldots, k$ occurs exactly once.

Let $\delta$ be a $k \times 1$ column vector, then following the notation used in (6), we shall denote by $P(\delta)$ the $q \times k(k-1)$ matrix obtained from $P$ on replacing the symbol $i$ by the element occurring in the $i$ th position in $\delta$. A similar meaning will be assigned to $P_{i}(\delta)$ and $\pi_{c j}(\delta)$ where $\pi_{c j}$ denotes the $j$ th column of $P_{c}$. If $D$ is a $k \times b$ matrix defined by

$$
D=\left[\delta_{1}, \delta_{2}, \ldots, \delta_{b}\right]
$$

where $\delta_{j}$ is a $k \times 1$ column vector, then we define $P(D)$ and $P_{i}(D)$ by

$$
\begin{aligned}
P(D) & =\left[P\left(\delta_{1}\right), P\left(\delta_{2}\right), \ldots, P\left(\delta_{b}\right)\right] \\
P_{i}(D) & =\left[P_{i}\left(\delta_{1}\right), P_{i}\left(\delta_{2}\right), \ldots, P_{i}\left(\delta_{b}\right)\right] .
\end{aligned}
$$

3. Main theorem. The theorem proved in this section is an improvement of the main theorem of (6).

Theorem 1. Let there exist a pairwise balanced design $(D)$ of index unity and type $\left(v ; k_{1}, k_{2}, \ldots, k_{m}\right)$ such that the set of equiblock components $\left(D_{1}\right),\left(D_{2}\right)$, $\ldots,\left(D_{l}\right), l<m$, is a clear set. If there exist $q_{i}-1$ mutually orthogonal Latin squares of order $k_{i}$ and if

$$
q^{*}=\min \left(q_{1}+1, \ldots, q_{l}+1, q_{l+1}, \ldots, q_{m}\right),
$$

then there exist at least $q^{*}-2$ mutually orthogonal Latin squares of order $v$.

Proof. Let us define

$$
q^{(1)}=\min \left(q_{1}+1, q_{2}+1, \ldots, q_{l}+1\right)
$$


and

$$
q^{(2)}=\min \left(q_{l+1}, q_{l+2}, \ldots, q_{m}\right)
$$

Then

$$
q^{*}=\min \left(q^{(1)}, q^{(2)}\right)
$$

Let

$$
\delta_{i 1}, \delta_{i 2}, \ldots, \delta_{i b_{i}}
$$

be the blocks of the equiblock component $\left(D_{i}\right)$ written out as columns $(i \leqslant l)$. By hypothesis there exist $q_{i}-1$ mutually orthogonal Latin squares of order $k_{i}$. Hence we can construct an orthogonal array $\mathrm{A}_{i j}$ with $q_{i}+1$ rows and $k_{i}{ }^{2}$ columns, whose symbols are the treatments occurring in $\delta_{i j}$. Let

$$
A_{i}=\left[A_{i 1}, A_{i 2}, \ldots, A_{i b_{i}}\right] \text {. }
$$

Let $\Delta_{i}$ be the $q^{*} \times b_{i} k_{i}{ }^{2}$ matrix obtained from $A_{i}$ by retaining only the first $q^{*}$ rows, and let

$$
\Delta^{(1)}=\left[\Delta_{1}, \Delta_{2}, \ldots, \Delta_{l}\right] .
$$

Then $\Delta^{(1)}$ has $q^{*}$ rows and $\sum b_{i} k_{i}{ }^{2}$ columns. Clearly $\Delta^{(1)}$ has the property that if $t_{c}$ and $t_{d}$ are any two treatments identical or distinct contained in any block of $\left(D_{1}\right),\left(D_{2}\right), \ldots$, or $\left(D_{l}\right)$, then the ordered pair $t_{c}, t_{d}$ occurs as a column exactly once in any two-rowed submatrix of $\Delta^{(1)}$.

Let $\Delta_{u}$ be the matrix obtained from $P_{u}\left(D_{u}\right)$ by retaining only the first $q^{*}$ rows, $u=l+1, \ldots, m$. Then

$$
\Delta^{(2)}=\left[\Delta_{l+1}, \Delta_{l+2}, \ldots, \Delta_{m}\right]
$$

has the property that if $t_{a}$ and $t_{b}$ are any two distinct treatments contained in any block of $\left(D_{l+1}\right), \ldots,\left(D_{m}\right)$ then the ordered pair $t_{a}, t_{b}$ occurs exactly once in any two-rowed submatrix of $\Delta^{(2)}$. The number of columns in $\Delta^{(2)}$ is

$$
\sum_{u=l+1}^{m} b_{u} k_{u}\left(k_{u}-1\right)
$$

Again let $\Delta^{(3)}$ be the $q^{*} \times v_{2}$ matrix whose $n$th column contains in every position the treatment $t_{n}$, where $t_{n}$ is any one of the

$$
v_{2}=v-\sum_{i=1}^{l} b_{i} k_{i}
$$

treatments not contained in $\left(D_{1}\right),\left(D_{2}\right), \ldots$, or $\left(D_{l}\right)$. Then $\left[\Delta^{(1)}, \Delta^{(2)}, \Delta^{(3)}\right]$ is an orthogonal array $\left(v^{2}, q^{*}, v, 2\right)$, and using any two rows for co-ordinatization we get $q^{*}-2$ mutually orthogonal Latin squares of order $v$.

4. Use of BIB designs. A balanced incomplete block (BIB) design with parameters $v, b, r, k, \lambda$ is an arrangement of $v$ objects or treatments into $b$ sets or blocks such that (i) each block contains $k<v$ different treatments, 
(ii) each treatment occurs in $r$ different blocks, and (iii) each pair of treatments occurs together in exactly $\lambda$ blocks. The parameters satisfy the relations

$$
\lambda(v-1)=r(k-1), b k=v r, b \geqslant v .
$$

These conditions are necessary but not sufficient for the existence of a BIB design. BIB designs were first introduced into statistical studies by Yates (15), but occur in earlier literature in connection with various combinatorial problems. Subsequent to Yates many authors have dealt with the problem of constructing these designs. Without attempting a complete bibliography we shall only refer to (1). A BIB design is said to be symmetric if $v=b$, and in consequence $k=r$. A BIB design is said to be resolvable (2) if the blocks can be divided into sets, such that the blocks of a given set contain each treatment exactly once. A resolvable or a symmetric BIB design is evidently separable.

A BIB design with $\lambda=1$ is clearly a pairwise balanced design of index unity and type $(v ; k)$. We shall denote such a design by BIB $(v ; k)$.

By omitting a single treatment from the design BIB $(v ; k)$ we get a pairwise balanced design of index unity and type $(v-1 ; k, k-1)$ where the $r$ blocks of size $k-1$ form a clear set. Again if from a BIB $(v ; k)$ we delete $x$ treatments belonging to the same block, $2 \leqslant x \leqslant k$, we get a pairwise balanced design of index unity and type $(v-x ; k, k-1, k-x)$ where the equiblock component consisting of the single block of size $k-x$ is clear. Hence we have

Theorem 2. Existence of a BIB (v;k) implies

(i) $N(v-1) \geqslant \min (N(k), 1+N(k-1))-1$,

(ii) $N(v-x) \geqslant \min (N(k), N(k-1), 1+N(k-x))-1$, if $2 \leqslant x \leqslant k$.

Example (1). Consider the BIB design with parameters $v=b=s^{2}+s+1$, $r=k=s+1, \lambda=1$, with $s=16$. Taking $x=6,8,9$ respectively we get $N(267) \geqslant 10, N(265) \geqslant 8, N(264) \geqslant 7$, whereas $n(267)=2, n(265)=4$ and $n(264)=2$.

Suppose we omit three treatments $\alpha_{1}, \alpha_{2}, \alpha_{3}$ not occurring in the same block of a BIB $(v ; k)$, then we get a pairwise balanced design $(D)$ of index unity and type $(v-3 ; k, k-1, k-2)$. Since in the original BIB $(v ; k)$ no two blocks can have more than one treatment in common, the three blocks of $(D)$ of size $k-2$ which have been obtained by deleting $\left(\alpha_{1}, \alpha_{2}\right),\left(\alpha_{2}, \alpha_{3}\right)$, $\left(\alpha_{1}, \alpha_{3}\right)$ have obviously no treatment in common and form a clear equiblock component. Hence we get

Theorem 3. The existence of a BIB $(v ; k)$ implies that

$$
N(v-3) \geqslant \min (N(k), N(k-1), 1+N(k-2))-1 .
$$

Example (2). Consider the BIB $(v ; k)$ designs (1, pp. 386-389) with $k=5$ and $v=21,25,41,45,61,65,85,125$. It follows that there exist at least two m.o.l.s. of the following orders: $18,22,38,42,58,62,82$, and 122 . 
Example (3). From the designs BIB $(81 ; 9)$ and BIB $(73 ; 9)$ we get $N(78) \geqslant 6, N(70) \geqslant 6$.

Example (4). From the design BIB $(273 ; 17)$ we get $N(270) \geqslant 2$ since $N(15) \geqslant n(15)=2$.

Suppose there exists a resolvable BIB design with parameters $v, b, r, k$, $\lambda=1$. Let $1<x \leqslant r$. To each block of the $i$ th replication add a new treatment $\theta_{i}, i=1,2, \ldots, x$, and add a new block $\theta_{1}, \theta_{2}, \ldots, \theta_{x}$. We then get a pairwise balanced design of index unity and type $(v+x ; k+1, k, x)$ if $x<r$, and type $(v+x ; k+1, r)$ if $x=r$. The equiblock component formed by the new block is clear. When $x=r-1$, the set of equiblock components consisting of the new block, and the blocks of the $r$ th replication is a clear set. Again by adding a treatment $\theta$ to all the blocks of a single replication we get $(v+1$; $k+1, k)$. Hence we have

Theorem 4A. The existence of a resolvable BIB $(v ; k)$ implies

(i) $N(v+x) \geqslant \min (N(k), N(k+1), 1+N(x))-1$ if $1<x \leqslant r-2$,

(ii) $N(v+r-1) \geqslant \min (1+N(k), N(k+1), 1+N(r-1))-1$,

(iii) $N(v+r) \geqslant \min (N(k+1), 1+N(r))-1$,

(iv) $N(v+1) \geqslant \min (N(k), N(k+1))-1$.

Example (5). Taking $x=5$ in the BIB design with $v=49, b=56, r=8$, $k=7, \lambda=1$, we have

$$
N(54) \geqslant \min (N(7), N(8), 1+N(5))-1=4 .
$$

Example (6). Using the resolvable BIB design $v=21, b=70, r=10$, $k=3, \lambda=1$, (5, p. 171, Table III) we have from part (ii) of the theorem $N(30) \geqslant 2$.

Again suppose there exists a separable BIB $(v ; k)$ in which the blocks can be divided into $n$ sets of type I. The number of replications is $r=k n$. For a symmetric design $n=1$. Let the blocks be written out as columns, and let $\left(S_{j}\right)$ be the $j$ th subset, the blocks being in the standard form $(j=1,2$, $\ldots, n)$. Let us take $r$ new treatments $\theta_{i j}, i=1,2, \ldots, k ; j=1,2, \ldots, n$. Let us define the $1 \times v$ row vector $\theta_{i j}=\left(\theta_{i j}, \theta_{i j}, \ldots, \theta_{i j}\right)$. Then we can denote by

$$
\left(\begin{array}{l}
S_{j} \\
\Theta_{i j}
\end{array}\right)
$$

the result of adding $\theta_{i j}$ in the $(k+1)$ th position to each block of the $j$ th subset.

Let $N(k+1)=q^{(1)}-1$. Then we can construct a $q^{(1)} \times(k+1) k$ matrix $P^{(1)}$ with the properties (i) and (ii) of Lemma 2 , where $P_{1}^{(1)}, P_{2}{ }^{(1)}, \ldots, P_{k}^{(1)}$ are the submatrices referred to in part (ii). If $\delta_{j u}$ is the $u$ th block of $\left(S_{j}\right)$, $u=1,2, \ldots, v$, then the corresponding block of

$$
\left(\begin{array}{c}
S_{j} \\
\Theta_{i j}
\end{array}\right)
$$


is

$$
\left(\begin{array}{c}
\delta_{j u} \\
\theta_{i j}
\end{array}\right)
$$

Consistent with our notation we can denote by

$$
P_{i}^{(1)}\left(\begin{array}{c}
\delta_{j u} \\
\theta_{i j}
\end{array}\right)
$$

the result of replacing the symbols $1,2, \ldots, k, k+1$ in $P_{i}^{(1)}$ by treatments in the 1 st, $2 \mathrm{nd}, \ldots,(k+1)$ th position in

$$
\left(\begin{array}{c}
\delta_{j u} \\
\theta_{i j}
\end{array}\right)
$$

and define

$$
P_{i}^{(1)}\left(\begin{array}{c}
S_{j} \\
\Theta_{i j}
\end{array}\right) \equiv\left[P_{i}^{(1)}\left(\begin{array}{c}
\delta_{j 1} \\
\theta_{i j}
\end{array}\right), \ldots, P_{i}^{(1)}\left(\begin{array}{c}
\delta_{j v} \\
\theta_{i j}
\end{array}\right)\right] .
$$

A pair of distinct treatments belonging to the original BIB design may be called a pure pair. Again a pair of treatments, one of which belongs to the original BIB design, and the other to the newly added treatments, may be called a mixed pair. Then

$$
\Delta_{1}=\left[\ldots, P_{i}^{(1)}\left(\begin{array}{c}
S_{j} \\
\Theta_{i j}
\end{array}\right), \ldots\right], i=1,2, \ldots, k ; j=1,2, \ldots, n
$$

has the property that any two-rowed submatrix contains as a column each pure and each mixed ordered pair of treatments exactly once.

Again if $q^{(2)}-1=N(r)$, we can form an orthogonal array $\Delta_{2}=\left(r^{2}, q^{(2)}+1\right.$, $r, 2)$ whose symbols are the $r$ new treatments. Let

$$
q=\min \left(q^{(1)}, q^{(2)}+1\right)
$$

and let $\Delta^{(1)}$ and $\Delta^{(2)}$ be obtained from $\Delta_{1}$ and $\Delta_{2}$ respectively by retaining the first $q$ rows only. Also let $\Delta^{(3)}$ be the $q \times v$ matrix whose $u$ th column contains the $u$ th treatment of the BIB design in each position. Then

$$
\Delta=\left[\Delta^{(1)}, \Delta^{(2)}, \Delta^{(3)}\right]
$$

is an orthogonal array of size $(v+r)^{2}, q$ constraints, $v+r$ levels and strength 2 , and is therefore equivalent to a set of $q-2$ mutually orthogonal Latin squares. Hence we have

THEOREM 4B. If there exists a $\mathrm{BIB}(v ; k)$ with $r$ replications, in which the blocks can be subdivided into sets of type $I$, then

$$
N(v+r) \geqslant \min (N(k+1), 1+N(r))-1 .
$$

Example (7). The existence of symmetric $\operatorname{BIB}(7 ; 3)$ and BIB $(57 ; 8)$ implies $N(10) \geqslant 2$ and $N(65) \geqslant 7$. 
Example (8). There exists a BIB design (1, p. 383) with parameters $v=25$, $b=50, r=8, k=4, \lambda=1$ for which the blocks can be separated into two subsets of type I. It follows that $N(33) \geqslant 3$.

Theorems $4 \mathrm{~A}$ and $4 \mathrm{~B}$ are special cases of the following more general theorem, the proof of which can be given on analogous lines.

THEOREM 4. If there exists a separable BIB $(v ; k)$ with $n_{1}$ subsets of type I and $n_{2}$ subsets of type II, so that the number of replications is $r=k n_{1}+n_{2}$, then

(i) $N(v+x) \geqslant \min (N(k), N(k+1), 1+N(x))-1$, if $x=k r_{1}+r_{2}$; $r_{1} \leqslant n_{1}, r_{2} \leqslant n_{2} ; 1<x<r-1$.

(ii) $N(v+r-1) \geqslant \min (1+N(k), N(k+1), 1+N(r-1))-1$ if $n_{2}>0$.

(iii) $N(v+r) \geqslant \min (N(k+1), 1+N(r))-1$.

(iv) $N(v+1) \geqslant \min (N(k+1), N(k))-1$ if $n_{2}>0$.

5. Use of GD designs. An arrangement of $v$ objects (treatments) in $b$ sets (blocks) each containing $k$ distinct treatments is said to be a group divisible (GD) design if the treatments can be divided into $l$ groups of $m$ treatments each, so that any two treatments belonging to the same group occur together in $\lambda_{1}$ blocks, and any two treatments from different groups occur together in $\lambda_{2}$ blocks. We will denote such a design by the notation GD $\left(v ; k, m ; \lambda_{1}, \lambda_{2}\right)$. The combinatorial properties of these designs have been studied in (3) where it has been shown that

$$
v=l m, b k=v r, \lambda_{1}(m-1)+\lambda_{2} m(l-1)=r(k-1),
$$

$r$ being the number of replications, that is, the number of times each treatment occurs in the design. It has also been shown that

$$
P=r k-\lambda_{2} v \geqslant 0, \quad Q=r-\lambda_{1} \geqslant 0 .
$$

The GD designs can be divided into three classes.

(i) Regular (R) characterized by $P>0, Q>0$.

(ii) Semi-regular (SR) characterized by $P=0, Q>0$.

(iii) Singular (S) characterized by $Q=0$.

Methods of constructing these designs have been given in (5). So far as the construction of mutually orthogonal Latin squares is concerned a special role is played by GD designs with $\lambda_{1}=0, \lambda_{2}=1$, which in our notation can be denoted by $\operatorname{GD}(v ; k, m ; 0,1)$. If, further, this design is regular we shall denote it by $\operatorname{RGD}(v ; k, m ; 0,1)$ and if it is semi-regular we shall denote it by $\operatorname{SRGD}(v ; k, m ; 0,1)$.

If to the $b$ blocks of the GD design with $\lambda_{1}=0, \lambda_{2}=1$, we add $l$ new blocks corresponding to the groups, we get a pairwise balanced design of index unity and type $(v ; k, m)$. The blocks of size $m$ form a clear equiblock component. Hence we have

TheOREM 5. If there exists a $\mathrm{GD}(v ; k, m ; 0,1)$ then

$$
N(v) \geqslant \min (N(k), 1+N(m))-1 .
$$


Corollary. $N\left(s^{2}-1\right) \geqslant N(s-1)$, if $s$ is a prime power.

This follows from the existence of a resolvable $\operatorname{GD}\left(s^{2}-1 ; s, s-1 ; 0,1\right)$.

Theorem 6. If there exists a $\mathrm{GD}(v ; k, m ; 0,1)$ then

$$
N(v-1) \geqslant \min (N(k), N(k-1), 1+N(m), 1+N(m-1))-1,
$$

and if the design is resolvable then

$$
N(v-1) \geqslant \min (N(k), N(k-1), N(m), N(m-1)) .
$$

The first part follows from the fact that if we omit any particular treatment from the corresponding pairwise balanced design of index unity and type $(v ; k, m ; 0,1)$ we get a design of the type $(v-1 ; k, k-1 ; m, m-1)$, in which the equiblock components with blocks of sizes $m$ and $m-1$ form a clear set. The second part has already been proved in (6) and is given here for completeness.

Theorem 7 . Suppose there exists a resolvable $\mathrm{GD}(v ; k, m ; 0,1)$ with $r$ replications, then

(i) $N(v+1) \geqslant \min (N(k), N(k+1), 1+N(m))-1$,

(ii) $N(v+x) \geqslant \min (N(k), N(k+1), 1+N(m), 1+N(x))-1$ if $1<x<r$

(iii) (a) $N(v+r) \geqslant \min (N(k+1), 1+N(m), 1+N(r))-1$, (b) $N(v+r) \geqslant \min (N(k+1), N(m+1), 1+N(k), 1+N(r))-1$,

(iv) $N(v+r+1) \geqslant \min (N(k+1), N(m+1), 1+N(r+1))-1$, where in part (iii) we choose whichever lower bound is better for $N(v+r)$.

To prove part (i) we add a new treatment $\theta_{1}$ to each block of one replication. To prove part (ii) we add a new treatment $\theta_{i}$ to each block of the $i$ th replication, $i=1,2, \ldots, x$, and take a new block $\left(\theta_{1}, \theta_{2}, \ldots, \theta_{x}\right)$. For the first part we note that the equiblock component given by the groups forms a clear set. For the second part we note that the set of equiblock components given by the groups and the new block is a clear set. To prove part (iii) (a) we add a new treatment $\theta_{i}$ to each block of the $i$ th replication, $i=1,2, \ldots$, $r$, and a new block $\left(\theta_{1}, \theta_{2}, \ldots, \theta_{r}\right)$. To prove part (iii) (b) we add a new treatment $\theta_{i}$ to each block of the $i$ th replication for the first $r-1$ replications, and a new treatment $\theta_{0}$ to each of the groups, and add a new block $\left(\theta_{0}, \theta_{1}, \ldots\right.$, $\left.\theta_{r-1}\right)$. We note in this case that the set of equiblock components given by the $r$ th replication, and the newly added block is a clear set. To prove (iv) we add one new treatment to the blocks of each replication, one new treatment to each of the blocks corresponding to the groups, and take a block containing the new treatments.

The group designs most useful to us are the semi-regular group divisible designs with $\lambda_{1}=0, \lambda_{2}=1$. For such a design the number of replications $r$ is equal to the group size $m$, and $v=k m$ In the notation used in (6) such a design is denoted by $\operatorname{SRGD}(k m ; k, m ; 0,1)$. It is known (5) that for an 
SRGD design each block contains the same number of treatments from each group. We shall now prove

Lemma 3. There exists a resolvable SRGD $(k m ; k, m ; 0,1)$ if $k \leqslant N(m)+1$. For this design the block size is $k, r=m$, and $b=m^{2}$.

Applying Lemma 2 we find that there exists a matrix $P$ with $N(m)+1$ rows and $m(m-1)$ columns such that in every two-rowed submatrix of $P$ every ordered pair of distinct symbols occurs exactly once, and it can be subdivided into $m-1$ parts such that in each row of every part every symbol occurs once. Let $P_{k}$ be the matrix obtained from $P$ by retaining only $k$ rows. Let $E_{k}$ be a $k \times m$ matrix such that the $i$ th column contains the $i$ th symbol in each position. Let $\Delta_{k}=\left[E_{k}, P_{k}\right]$. Now let us consider $k m$ treatments $t, t_{2}, \ldots, t_{k m}$. Let the symbols $1,2, \ldots, m$ in the $j$ th row of $\Delta_{k}$ be replaced by

$$
t_{\alpha+1}, t_{\alpha+2}, \ldots, t_{\alpha+m} \text { where } \alpha=(j-1) m .
$$

This gives an SRGD with the required properties. The blocks are given by the columns. The replications consist of $E_{k}$ and subdivisions of $P_{k}$.

Combining Lemma 3 and Theorem 7 we have

Theorem 8. If $k \leqslant N(m)+1$, then

(i) $N(k m+1) \geqslant \min (N(k), N(k+1), 1+N(m))-1$,

(ii) $N(k m+x) \geqslant \min (N(k), N(k+1), 1+N(m), 1+N(x))-1$ if $1<x<m$.

Example (9). Taking $k, m$, and $x$ as shown we derive the lower bound for $N(k m+x)$, noting that $N(24) \geqslant 3$ from Table 1 of (6) and $N(10) \geqslant 2$ from Example (7), Theorem 4B.

$$
\begin{array}{llrl}
k=7, m=11, x=5 ; & N(82) \geqslant 4, \\
k=8, m=11, x=7 ; & N(95) \geqslant 6, \\
k=7, m=19, x=5 ; & N(138) \geqslant 4, \\
k=7, m=8, x=4 ; & N(60) \geqslant 3, \\
k=4, m=24, x=10 ; & N(106) \geqslant 2, \\
k=8, m=13, x=7 ; & N(111) \geqslant 6, \\
k=4, m=27, x=10 ; & N(118) \geqslant 2, \\
k=7, m=16, x=10 ; & N(122) \geqslant 2, \\
k=7, m=17, x=5 ; & N(124) \geqslant 4 .
\end{array}
$$

6. Use of the method of differences. Let $0,1,2, \ldots, n-1$ be the elements of the ring $R$ of residue classes $(\bmod n)$. We shall consider matrices whose elements belong either to $R$ or to the set $X$ of $m$ indefinites $x_{1}, x_{2}, \ldots, x_{m}$. We shall say that the difference associated with the ordered pair $\left(\begin{array}{l}i \\ j\end{array}\right)$, where $i$ and $j$ belong to $R$ is $c$ where $i-j \equiv c(\bmod n), 0 \leqslant c<n$. Conversely to each element $c$ of $R$ there correspond $n$ ordered pairs which have $c$ as their associated difference. If $\left(\begin{array}{l}i \\ j\end{array}\right)$ is one of these pairs then the other pairs are $\left(\begin{array}{l}i+\theta \\ j+\theta\end{array}\right)$ where $\theta=0,1,2, \ldots, n-1$, and $i+\theta$ and $j+\theta$ are reduced $(\bmod n)$. 
The ordered pair $\left(\begin{array}{l}i \\ j\end{array}\right)$ both members of which belong to $R$ will be called an $R$-pair. A pair $\left(\begin{array}{c}i \\ x_{j}\end{array}\right)$ where $i$ belongs to $R$ and $x_{j}$ to $X$ is called an $R X$-pair and the difference associated with it is defined to be $x_{j}$. If $\theta$ is any element of $R$ we shall formally define $x_{j}+\theta=x_{j}$. With this definition, corresponding to any indefinite $x_{j}$, there are $n R X$ pairs, the difference associated with each of which is $x_{j}$. If $\left(\begin{array}{c}i \\ x_{j}\end{array}\right)$ is one of these pairs then the other pairs are $\left(\begin{array}{c}i+\theta \\ x_{j}\end{array}\right)$ where $\theta=0,1, \ldots, n-1$. These pairs are of course all the pairs $\left(\begin{array}{c}i \\ x_{j}\end{array}\right), i=0,1, \ldots$, $n-1$ in some order or other. We may similarly define $X R$ pairs. The difference associated with the $X R$ pair $\left(\begin{array}{c}x_{i} \\ j\end{array}\right)$ is $x_{i}$.

We shall now prove the following theorem:

THEOREM 9. If $m$ is odd there exist at least two orthogonal Latin squares of order $3 m+1$. Taking $m=4 t+3$ this implies the existence of a pair of orthogonal Latin squares for all orders $12 t+10$.

Consider the $4 \times 4 m$ matrix $A_{0}$ given below (to exhibit the structure of $\mathrm{A}_{0}$ it is divided into 4 parts), whose elements belong to $R$ the ring of residue classes $\bmod (2 m+1)$ or $X$ the set of indefinites $x_{1}, x_{2}, \ldots, x_{m}$.

$$
\begin{aligned}
& \mathrm{A}_{0}=\left[\begin{array}{llllllll}
0 & 0 & \ldots & 1 & 2 & \ldots m & 2 m 2 m-1 \ldots m+1 \\
1 & 2 & \ldots m & 0 & 0 & \ldots 0 & x_{1} x_{2} & \ldots x_{m} \\
2 m & 2 m-1 \ldots m+1 & x_{1} & x_{2} & \ldots x_{m} & 0 & 0 & \ldots 0 \\
x_{1} & x_{2} & \ldots x_{m} & 2 m & 2 m-1 \ldots m+1 & 1 & 2 & \ldots m
\end{array}\right]
\end{aligned}
$$

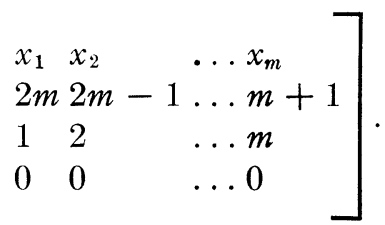

We note that of the $4 m$ pairs occurring as columns in any two-rowed submatrix of $A_{0}, 2 m$ are $R$-pairs, the differences associated with which are all the non-null elements of $R, m$ are $R X$-pairs the differences associated with which are all the elements of $X$ and the same is true of $X R$ pairs. Let $A_{\theta}$ be the matrix derived from $A_{0}$ by adding $\theta, 0 \leqslant \theta \leqslant 2 m$, to every element of $A_{0}$ and reducing $\bmod (2 m+1) ; x_{i}+\theta$ being considered as $x_{i}$. Let

$$
A=\left[A_{0}, A_{1}, \ldots, A_{2 m}\right] \text {. }
$$

Then it is evident that in any two-rowed submatrix of $A$, any $R$-pair formed by two distinct elements of $R$, or any $R X$ or $X R$-pair occurs exactly once. Let $A^{*}$ be an orthogonal array $\left[m^{2}, 4, m, 2\right]$ corresponding to two orthogonal Latin squares formed by the symbols $x_{1}, x_{2}, \ldots, x_{m}$, and let $E$ be a $4 \times(2 m+1)$ matrix whose $i$ th column contains $i$ in each place $(0 \leqslant i \leqslant 2 m)$. Then

$$
\Delta=\left[E, A, A^{*}\right]
$$

is an orthogonal array $\left[(3 m+1)^{2}, 4,3 m+1,2\right]$, which proves the result. 
Example (10). Taking $t=0,1,2,3,4,8,9$, and 12 respectively it follows that $N(v) \geqslant 2$ for $v=10,22,34,46,58,106,118$, and 154 .

Two superposed $10 \times 10$ orthogonal squares obtained by this method are exhibited below. The symbols $x_{1}, x_{2}, x_{3}$ have been replaced by $7,8,9$.

\section{A Pair of $10 \times 10$ Orthogonal Souares}

$\begin{array}{llllllllll}00 & 67 & 58 & 49 & 91 & 83 & 75 & 12 & 24 & 36 \\ 76 & 11 & 07 & 68 & 59 & 92 & 84 & 23 & 35 & 40 \\ 85 & 70 & 22 & 17 & 08 & 69 & 93 & 34 & 46 & 51 \\ 94 & 86 & 71 & 33 & 27 & 18 & 09 & 45 & 50 & 62 \\ 19 & 95 & 80 & 72 & 44 & 37 & 28 & 56 & 61 & 03 \\ 38 & 29 & 96 & 81 & 73 & 55 & 47 & 60 & 02 & 14 \\ 57 & 48 & 39 & 90 & 82 & 74 & 66 & 01 & 13 & 25 \\ 21 & 32 & 43 & 54 & 65 & 06 & 10 & 77 & 88 & 99 \\ 42 & 53 & 64 & 05 & 16 & 20 & 31 & 89 & 97 & 78 \\ 63 & 04 & 15 & 26 & 30 & 41 & 52 & 98 & 79 & 87\end{array}$

We shall now give two special examples of the use of the method of differences. Example (11). Consider the matrix

$$
P_{0}=\left[\begin{array}{llll}
0 & x_{1} & x_{2} & x_{3} \\
1 & 0 & 0 & 0 \\
4 & 4 & 6 & 9 \\
6 & 1 & 2 & 8
\end{array}\right]
$$

whose elements belong to the ring $R$ of residue classes $(\bmod 11)$ and the set $X$ of indefinites $x_{1}, x_{2}, x_{3}$. Let $P_{1}, P_{2}, P_{3}$ be obtained from $P_{0}$ by cyclic permutation of the rows, and let

$$
A_{0}=\left[P_{0}, P_{1}, P_{2}, P_{3}\right] \text {. }
$$

Then it is easy to verify that each two-rowed submatrix of $A_{0}$ contains as columns $10 R$-pairs, the differences associated with which are all the nonnull elements of $R ; 3 R X$-pairs the differences associated with which are the 3 elements of $X$, and $3 X R$ pairs for which the same is true. Let $A_{\theta}$ be the matrix obtained from $A_{0}$ by adding $\theta$ to elements of $A_{0}$, where $\theta$ belongs to $R$. Then

$$
A=\left[A_{0}, A_{1}, \ldots, A_{10}\right]
$$

is a matrix such that any two-rowed submatrix contains as a column every $R$-pair consisting of distinct elements of $R$, and every $R X$ and $X R$-pair, exactly once. If $A^{*}$ is the orthogonal array of strength 2 and 4 constraints with the symbols $x_{1}, x_{2}, x_{3}$ and $E$ is the $4 \times 11$ matrix for which the $i$ th column contains $i$ in every place $(i=0,1, \ldots, 10)$ then

$$
\Delta=\left[E, A, A^{*}\right]
$$


is an orthogonal array $\left[14^{2}, 4,14,2\right]$ from which a pair of orthogonal Latin squares of order 14 can be constructed.

Example (12). Similarly by starting with the matrix

$$
P_{0}=\left[\begin{array}{rrrrccc}
0 & 0 & 0 & 0 & x_{1} & x_{2} & x_{3} \\
3 & 6 & 2 & 1 & 0 & 0 & 0 \\
8 & 20 & 12 & 16 & 20 & 17 & 8 \\
12 & 16 & 7 & 2 & 19 & 6 & 21
\end{array}\right]
$$

whose elements belong to the ring $R$ of residue classes $(\bmod 23)$ and the set $X$ of indefinites $x_{1}, x_{2}, x_{3}$, we can construct two mutually orthogonal Latin squares of order 26 .

7. Improved lower bounds for $N(v), v \leqslant 154$. We give here those values of $v \leqslant 154$ for which the lower bound of $N(v)$ can be improved over the bound given in Table I of (6).

TABLE I

\begin{tabular}{|c|c|c|c|}
\hline$v$ & $n(v)$ & $\begin{array}{l}\text { 1.b. for } \\
N(v)\end{array}$ & Remarks \\
\hline 10 & 1 & 2 & Ex. (7), Th. 4 B or Ex. (10), Th. 9 \\
\hline 14 & 1 & 2 & Ex. (11) \\
\hline 18 & 1 & 2 & Ex. (2), Th. 3 \\
\hline 26 & 1 & 2 & Ex. (12) \\
\hline 30 & 1 & 2 & Ex. (6), Th. $4 \mathrm{~A}$ \\
\hline 33 & 2 & 3 & Ex. (8), Th. 4B \\
\hline 34 & 1 & 2 & Ex. (10), Th. 9 \\
\hline 38 & 1 & 2 & Ex. (2), Th. 3 \\
\hline 42 & 1 & 2 & Ex. (2), Th. 3 \\
\hline 46 & 1 & 2 & Ex. (10), Th. 9 \\
\hline 54 & 1 & 4 & Ex. (5), Th. $4 \mathrm{~A}$ \\
\hline 60 & 2 & 3 & Ex. (9) (iv), Th. 8 \\
\hline 62 & 1 & 2 & Ex. (2), Th. 3 \\
\hline 65 & 4 & 7 & Ex. (7), Th. $4 \mathrm{~B}$ \\
\hline 70 & 1 & 6 & Ex. (3), Th. 3 \\
\hline 78 & 1 & 6 & Ex. (3), Th. 3 \\
\hline 82 & 1 & 4 & Ex. (9) (i), Th. 8 \\
\hline 90 & 1 & 2 & $90=10 \times 9$ and Lemma 1 \\
\hline 95 & 4 & 6 & Ex. (9) (ii), Th. 8 \\
\hline 106 & 1 & 2 & Ex. (10), Th. 9, or Ex. (9) (v), Th. 8 \\
\hline 111 & 2 & 6 & Ex. (9) (vi), Th. 8 \\
\hline 114 & 1 & 2 & $114=38 \times 3$ and Lemma 1 \\
\hline 118 & 1 & 2 & Ex. (10), Th. 9, or Ex. (9) (vii), Th. 8 \\
\hline 122 & 1 & 2 & Ex. (2), Th. 3 , or Ex. (9) (viii), Th. 8 \\
\hline 124 & 3 & 4 & Ex. (9) (ix), Th. 8 \\
\hline 138 & 1 & 4 & Ex. (9) (iii), Th. 8 \\
\hline 154 & 1 & 2 & Ex. (10), Th. 9 , or $154=22 \times 7$ and Lemma 1 \\
\hline
\end{tabular}




\section{The existence of at least two orthogonal Latin squares of order} $v>6$. If $v$ is divisible by 4 or if $v$ is odd then $N(v) \geqslant n(v) \geqslant 2$. Hence we need only consider numbers for which $v \equiv 2(\bmod 4)$. We shall first prove

LEMMA 4. $N(v) \geqslant 2$ if $6<v \leqslant 726$.

This result has already been checked in Table I of (6), supplemented by the improvements noted in Table I of the last section, up to $v=154$.

Any integer $v$ lying in the closed interval $I_{i}=\left(a_{i}, b_{i}\right)$ shown in column (2) of Table II can be expressed in the form

$$
v=4 m_{i}+x_{i}, \quad 10 \leqslant x_{i} \leqslant c_{i}
$$

where $m_{i}$ and $c_{i}$ are given in columns (3) and (4), since $a_{i}=4 m_{i}+10$, $b_{i}=4 m_{i}+c_{i}$.

TABLE II

\begin{tabular}{ccrc}
\hline$i$ & Interval $I_{i}=\left(a_{i}, b_{i}\right)$ & $m_{i}$ & $c_{i}$ \\
\hline 1 & $(158,182)$ & 37 & 34 \\
2 & $(186,218)$ & 44 & 42 \\
3 & $(222,262)$ & 53 & 50 \\
4 & $(266,310)$ & 64 & 54 \\
5 & $(314,374)$ & 76 & 70 \\
6 & $(378,454)$ & 92 & 86 \\
7 & $(458,550)$ & 112 & 102 \\
8 & $(554,662)$ & 136 & 118 \\
9 & $(666,726)$ & 164 & 70 \\
\hline
\end{tabular}

It is readily verified that $N\left(m_{i}\right) \geqslant n\left(m_{i}\right) \geqslant 3$. Again $N\left(x_{i}\right) \geqslant 2$ since $10 \leqslant x_{i} \leqslant c_{i}<154$. If we take $k=4$ in part (ii) of Theorem 8 , the conditions $k \leqslant N\left(m_{i}\right)+1$ and $1<x_{i}<m_{i}$ are obviously satisfied. Hence if $v$ lies in any of the closed intervals $I_{i}(i=1,2, \ldots, 9), N(v) \geqslant 2$. The Lemma follows by noting that any $v \equiv 2(\bmod 4)$ and satisfying $154<v \leqslant 726$ lies in one of the closed intervals $I_{i}$.

THEOREM 10. There exist at least two orthogonal Latin squares of any order $v>6$.

It is sufficient to prove the theorem for numbers $v \equiv 2(\bmod 4), v \geqslant 730$. If $v$ satisfies these conditions we can write

$$
v-10=144 g+4 u, \quad g \geqslant 5, \quad 0 \leqslant u \leqslant 35
$$

therefore

$$
v=4(36 g)+4 u+10 .
$$

Since the least factor in the prime power decomposition of $36 \mathrm{~g}$ is necessarily greater than or equal to $4, N(36 g) \geqslant n(36 g) \geqslant 3$. If in Theorem 8 part (ii) 
we take $k=4, m=36 g, x=4 u+10$, then $k \leqslant 1+N(m)$. Also $10 \leqslant x \leqslant 150$, $m \geqslant 180$. Hence $1<x<m$, and $N(x) \geqslant 2$. It follows that $N(v) \geqslant 2$.

The question raised in the concluding remarks of (6) is thus completely answered. If a positive integer $v>2$ is called Eulerian if two orthogonal Latin squares of order $v$ do not exist, then 6 is the only Eulerian number.

\title{
REFERENCES
}

1. R. C. Bose, On the construction of balanced incomplete block designs, Ann. Eugen. London, 9 (1939), 353-399.

2. - A note on the resolvability of balanced incomplete block designs, Sankhyā, 6 (1942), $105-110$.

3. R. C. Bose and W. S. Connor, Combinatorial properties of group divisible incomplete block designs, Ann. Math. Stat., 23 (1952), 367-383.

4. R. C. Bose and S. S. Shrikhande, On the falsity of Euler's conjecture about the non-existence of two orthogonal Latin squares of order $4 t+2$, Proc. Nat. Acad. Sci. U.S.A., 45 (1959), $734-737$.

5. R. C. Bose, S. S. Shrikhande, and K. Bhattacharya, On the construction of group divisible incomplete block designs, Ann. Math. Stat., 24 (1953), 167-195.

6. - On the construction of pairwise orthogonal Latin squares and the falsity of a conjecture of Euler, U. N. C., Institute of Statistics, Mimeo. series no. 222. To be published in Trans. Amer. Math. Soc.

7. K. A. Bush, Orthogonal arrays of index unity, Ann. Math. Stat., 28 (1952), 426-434.

8. L. Euler, Recherches sur une nouvelle espece des quarres magiques, Verh. zeeuwsch Genoot. Weten. Vliss., 9 (1782), 85-239.

9. F. W. Levi, Finite geometrical systems (University of Calcutta, 1942).

10. H. F. MacNeish, Euler squares, Ann. Math., 23 (1922), 221-227.

11. H. B. Mann, The construction of orthogonal Latin squares, Ann. Math. Stat., 13 (1942), 418-423.

12. E. T. Parker, Construction of some sets of pairwise orthogonal Latin squares, Amer. Math. Soc. Notices 5 (1958), 815 (abstract). (To be published in Proc. Amer. Math. Soc. under the title: Construction of some sets of mutually orthogonal Latin squares.)

13. - Orthogonal Latin squares, Proc. Nat. Acad. Sci. U.S.A., 45 (1959), 859-862.

14. C. R. Rao, Factorial experiments derivable from combinatorial arrangements of arrays, J. Roy. Stat. Soc. Suppl., 9 (1947), 128-139.

15. F. Yates, Incomplete randomised blocks, Ann. Eugen. London, 7 (1936), 121-140.

\author{
University of North Carolina, \\ Case Institute of Technology \\ and \\ Remington Rand Univac, St. Paul, Minnesota
}

